\title{
Gradient-Based Correction of Chromatic Aberration in the Joint Acquisition of Color and Near-Infrared Images
}

\author{
Zahra Sadeghipoor ${ }^{a}$, Yue M. Lu ${ }^{b}$, and Sabine Süsstrunk ${ }^{a}$ \\ ${ }^{a}$ School of Computer and Communication Sciences \\ École Polytechnique Fédérale de Lausanne (EPFL), Lausanne, Switzerland \\ ${ }^{b}$ Harvard School of Engineering and Applied Sciences, Cambridge, USA
}

\begin{abstract}
Chromatic aberration distortions such as wavelength-dependent blur are caused by imperfections in photographic lenses. These distortions are much more severe in the case of color and near-infrared joint acquisition, as a wider band of wavelengths is captured. In this paper, we consider a scenario where the color image is in focus, and the NIR image captured with the same lens and same focus settings is out-of-focus and blurred. To reduce chromatic aberration distortions, we propose an algorithm that estimates the blur kernel and deblurs the NIR image using the sharp color image as a guide in both steps. In the deblurring step, we retrieve the lost details of the NIR image by exploiting the sharp edges of the color image, as the gradients of color and NIR images are often correlated. However, differences of scene reflections and light in visible and NIR bands cause the gradients of color and NIR images to be different in some regions of the image. To handle this issue, our algorithm measures the similarities and differences between the gradients of the NIR and color channels. The similarity measures guide the deblurring algorithm to efficiently exploit the gradients of the color image in reconstructing high-frequency details of NIR, without discarding the inherent differences between these images. Simulation results verify the effectiveness of our algorithm, both in estimating the blur kernel and deblurring the NIR image, without producing ringing artifacts inherent to the results of most deblurring methods.
\end{abstract}

Keywords: Chromatic aberration, NIR imaging, blur kernel, gradient mask, guided image deblurring.

\section{INTRODUCTION}

We study the problem of axial chromatic aberration in the joint acquisition of color and near-infrared (NIR) images. Differences of light and scene reflections in the visible part of the electromagnetic spectrum (with wavelengths of 400-700 nm) and the NIR band (wavelength range of approximately 700-1100 nm) inspired researchers to use NIR information along with color images in tasks that are traditionally performed using color information only. Some of these applications are image denoising, ${ }^{1}$ image dehazing, ${ }^{2}$ shadow detection and removal, ${ }^{3,4}$ and video conference relighting. ${ }^{5}$

These recent developments introduce the need for designing a consumer camera that simultaneously captures both color (RGB) and NIR representations of the scene. The sensitivity of silicon-based sensors, placed in most current color cameras, goes beyond the visible range and extends to the NIR band as well (see Fig. 1). This enables designing a color and NIR camera with only a single sensor. ${ }^{6-8}$

Such an imaging system, similar to any single-sensor multispectral camera, would suffer from different aberration distortions introduced by the lens. One type of these distortions, called chromatic aberration (CA), occurs because the diffraction index of a simple lens changes with wavelength. As a result, light rays with different wavelengths are not all focused at the same point (Fig. 1-b). This causes the image of only one spectral channel to be sharp, whereas the others are blurred (see Fig. 2-a).

In color imaging, chromatic aberration distortions are reduced either by using multiple-element lenses and/or by post-processing the color channels. There exist professional lenses corrected for both visible and NIR bands of

Further author information: Send correspondence to Zahra Sadeghipoor, e-mail: zahra.sadeghipoor@epfl.ch, Telephone: +41216931274 


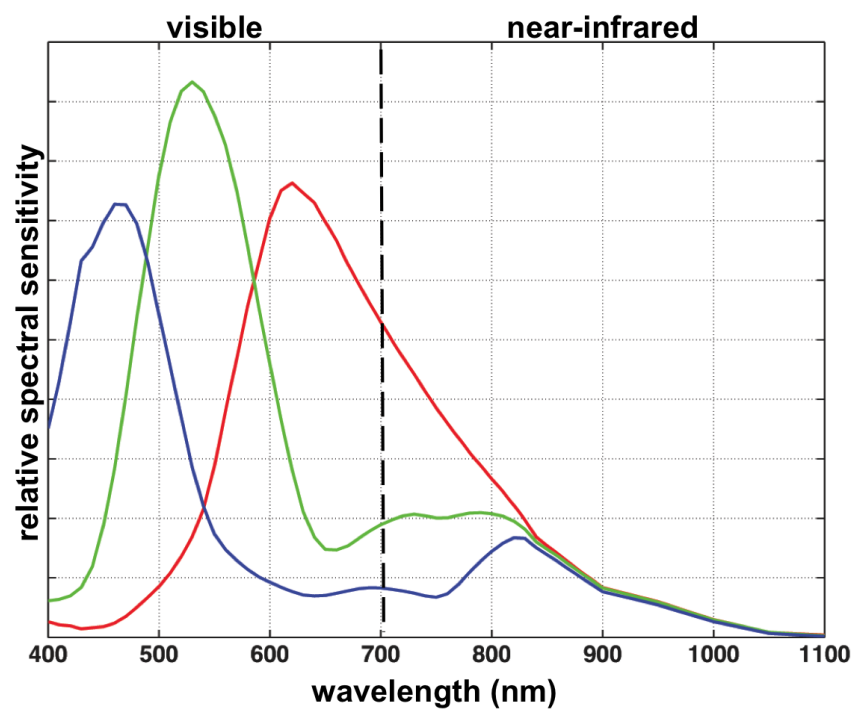

(a)

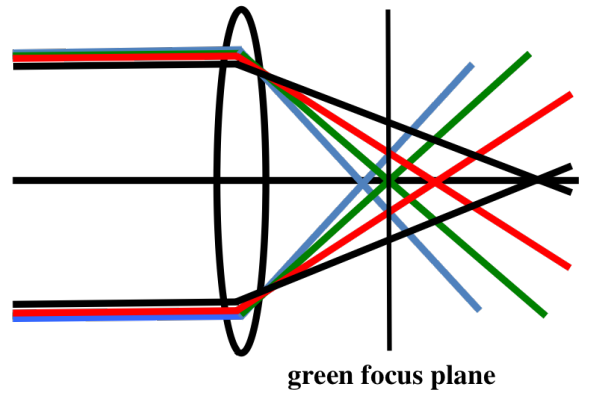

(b)

Figure 1. (a) The overall sensitivities of silicon sensor and color filters in a conventional color camera when the NIRblocking filter is removed (measured in our laboratory). The visible band is the wavelength range of 400 to approximately $700 \mathrm{~nm}$, and the NIR spectrum is adjacent to the visible band. In this paper, we define the NIR band to be the range of 700 to $1100 \mathrm{~nm}$, where silicon sensitivity approaches zero. (b) Axial chromatic aberration caused by a simple lens. If the sensor is placed at the focus point of the green light, the images of the red, blue, and, even more so, the NIR radiation are out-of-focus and blurred.

the spectrum*. However, such lenses are very expensive and too bulky to be used with most consumer cameras, especially those integrated inside small devices such as cellphones. Therefore, we are interested mainly in digital correction of CA after acquisition.

The algorithms developed to reduce the effects of $\mathrm{CA}$ in color imaging ${ }^{9-12}$ usually rely on the assumption that edges of color channels are highly correlated. Hence, it is possible to directly use the high-frequency components of the sharp channel in reconstructing the images of other channels. These algorithms cannot be employed in our problem. Firstly, because distortions caused by CA become more severe as the captured wavelength range increases. Hence, in the joint acquisition of color and NIR (wavelength range of 400-1100 nm) CA errors are much higher compared with distortions in color imaging (range of 400-700 nm). More importantly, as there are many differences between color and NIR channels, the main assumption of these algorithms is not valid in our task. Figure 2-(b) shows one example of the differences between the high-frequency details of color and NIR images.

In this paper, we assume that the lens is focused while capturing the color image ${ }^{\dagger}$, and the NIR image, which is recorded with the same focus settings, is blurred (see Fig. 2-a). We propose a two-step algorithm that reduces the axial chromatic aberration distortions noticeable in the NIR image in this case. The first step of our algorithm estimates the blur kernel by comparing the gradients of the blurred NIR channel and the sharp (in-focus) color image. The estimated blur kernel is then used to deblur NIR, guided by the color image. The main difficulty in deblurring is the fact that the differences of light and scene reflections in visible and NIR bands lead to differences between the gradients of color and NIR images, either in magnitude and/or direction (see Fig. 2-b). To account for these differences, we propose incorporating gradient masks in the deblurring step. Using the proposed masks ensures that the high-frequency components of the RGB image are used in deblurring NIR

\footnotetext{
${ }^{*}$ Example of a commercial product: http://www.jenoptik-inc.com/coastalopt-standard-lenses/uv-vis-nir-60mm-slrlens-mainmenu-155/80-uv-vis-ir-60-mm-apo-macro.html

${ }^{\dagger}$ Note that the axial chromatic aberration produced by most current lenses is negligible in color channels. See Fig. 2-(a).
} 

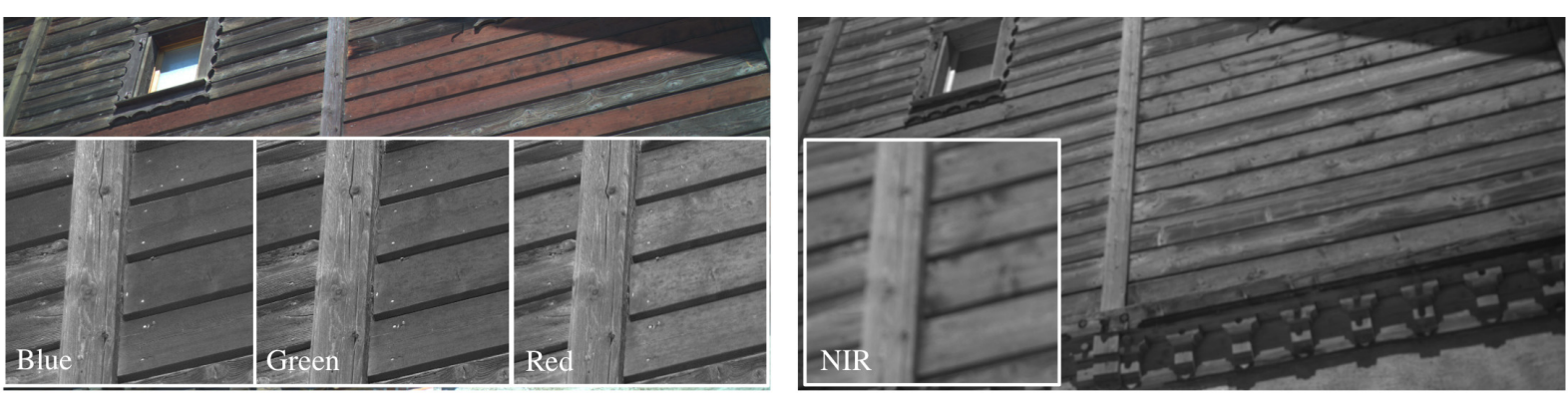

(a)
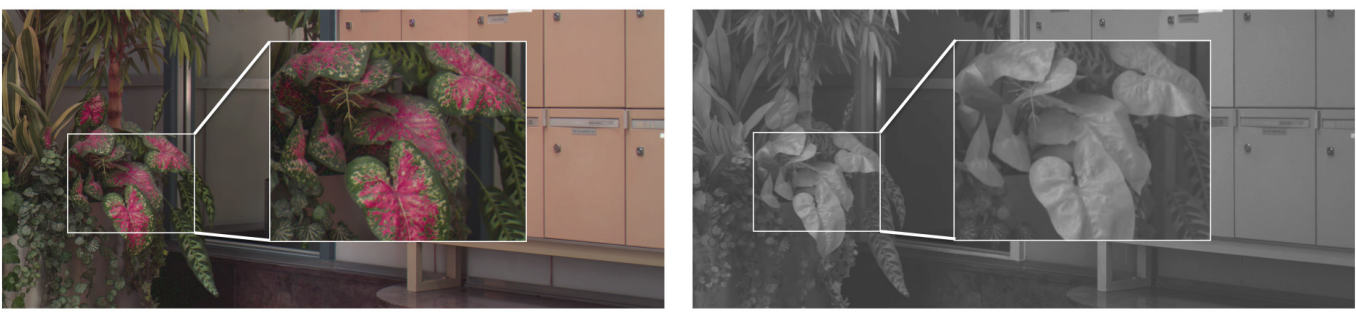

(b)

Figure 2. (a) A pair of RGB (left) and NIR (right) images captured with the same focus settings. The RGB image is in focus and sharp, while the NIR representation is blurred because of chromatic aberration. Insets: a part of the image in color and NIR channels (zoomed in). (b) A pair of color and NIR images. Insets show the region where color and NIR gradients are significantly different.

only where edges of these images are highly correlated. Otherwise, without the gradient masks, the deblurring process eliminates the inherent differences between color and NIR representations. This results in the failure of applications that fuse color and NIR information, such as dehazing, ${ }^{2}$ as many of these applications heavily rely on the intrinsic differences between the high-frequency details of these images. The details of computing these gradient masks are explained in the next section.

Many deblurring algorithms use only the blurred image to estimate a sharp representation of the scene. ${ }^{13-16}$ However, similar to our proposed algorithm, a few studies use a guide image in deblurring. He et al. ${ }^{17}$ propose the guided image filtering for the general problem of image restoration including image deblurring. The algorithm of Yuan et al. ${ }^{18}$ uses a noisy color image to guide the deblurring of a blurred color image of the same scene. Similarly, Zhang et al. ${ }^{19}$ propose using multiple blurred images of the scene to produce a sharp representation. The main difference between these algorithms and our work is that in their applications both guide and target images represent the scene in the visible band, hence their gradients are strongly correlated.

Yan et al. ${ }^{1}$ developed a guided denoising algorithm for situations where the gradients of the target and guide images are not necessarily the same. In the denoising task addressed in, ${ }^{1}$ the gradients of the input noisy image are not largely degraded compared with the target image. However, in our problem, the gradients of the input image (the blurred NIR) are significantly weaker than those of the guide (the color image). This, on top of the intrinsic differences between target and guide images, is potentially another source of confusion for a guided reconstruction approach. We explain how our algorithm handles this difficulty in the next section.

Simulation results, presented in Section 3, verify the effectiveness of our algorithm in recovering the details of the NIR image both when there is a strong correlation between color and NIR channels, and when the edges of these images are independent. We conclude the paper by discussing the results and possible directions for future research in Section 4. 


\section{THE PROPOSED ALGORITHM}

We mathematically formulate blurring of the NIR image as follows:

$$
\mathrm{NIR}_{b}=k * \mathrm{NIR},
$$

where NIR is the latent sharp image to be estimated, $k$ is the kernel point spread function (PSF) that characterizes the out-of-focus lens blur, $\mathrm{NIR}_{b}$ is the NIR image blurred because of chromatic aberration, and $*$ denotes the convolution operation.

In the following subsections, we explain the blur kernel estimation, and then we discuss how $\mathrm{NIR}_{b}$ is deblurred by exploiting the high-frequency details of the color image.

\subsection{Estimating the Blur Point Spread Function}

Let us denote the luminance channel of the color image, computed as the average of three color channels, by $Y$. We estimate the blur kernel $k$ by solving the following optimization problem:

$$
k^{\star}=\operatorname{argmin}\left\|\nabla_{x} \mathrm{NIR}_{b}-\nabla_{x}(k * Y)\right\|_{F}^{2}+\left\|\nabla_{y} \mathrm{NIR}_{b}-\nabla_{y}(k * Y)\right\|_{F}^{2}+\operatorname{Pr}(k),
$$

where $\nabla_{x}, \nabla_{y}$, respectively, represent the horizontal and vertical gradient operators. This optimization exploits the fact that, as opposed to absolute intensities, the edges of NIR and color channels are usually correlated (see Fig. 2-a). Note that color and NIR images do not always share similar edges, nevertheless, our experiments show that if there are some correlated edges in the image pair, the kernel estimated by solving the above problem is reliable.

$\operatorname{Pr}(k)$ in (2) represents the prior information about the kernel. The PSF of a lens is usually modeled as a Gaussian filter. ${ }^{20,21}$ Hence, we can write $k_{\sigma}=\exp \left(-\frac{x^{2}+y^{2}}{2 \sigma^{2}}\right)$, where $\sigma$ fully characterizes the kernel and is a measure of the filter's spread. In this paper, we assume that the blur kernel is spatially invariant. In this case, estimating the blur kernel is equivalent to finding one $\sigma$ value:

$$
\sigma^{\star}=\operatorname{argmin}\left\|\nabla_{x} \mathrm{NIR}_{b}-\nabla_{x}\left(k_{\sigma} * Y\right)\right\|_{F}^{2}+\left\|\nabla_{y} \mathrm{NIR}_{b}-\nabla_{y}\left(k_{\sigma} * Y\right)\right\|_{F}^{2} \quad \text { s.t. } k_{\sigma}=\frac{1}{c} \exp \left(-\frac{x^{2}+y^{2}}{2 \sigma^{2}}\right),
$$

where $c$ is a normalization factor ensuring that $\sum_{i}\left(k_{\sigma}\right)_{i}=1$.

\subsection{Deblurring the NIR Image}

As with the other non-blind deblurring methods, we formulate the NIR deblurring as solving the following problem:

$$
\mathrm{NIR}^{\star}=\operatorname{argmin}\left\|\mathrm{NIR}_{b}-k_{\sigma} * \mathrm{NIR}\right\|_{F}^{2}+\lambda \operatorname{Pr}(\mathrm{NIR}),
$$

where $k_{\sigma}$ is the blur kernel estimated in the first step (Section 2.1) and $\lambda$ is the regularization parameter.

Image deblurring is an ill-posed problem, hence including a regularization term $(\operatorname{Pr}(\mathrm{NIR}))$ to constrain the solution is necessary. Different prior terms are proposed in the deblurring literature. The most popular regularization term is the sparsity of gradients in natural images, usually modeled by Laplacian or hyperLaplacian distributions. ${ }^{22-24}$

In our scenario, however, the sharp RGB representation of the scene is available. Thus, instead of using a general distribution of natural images, we propose applying the following regularization term:

$$
\operatorname{Pr}(\mathrm{NIR})=\left\|\nabla_{x} \mathrm{NIR}-M_{x} \odot \nabla_{x} Y\right\|_{F}^{2}+\left\|\nabla_{y} \mathrm{NIR}-M_{y} \odot \nabla_{y} Y\right\|_{F}^{2} .
$$

Here, $M_{x}$ and $M_{y}$ are the gradient masks with the same size as the NIR and color images, and $\odot$ stands for element-wise multiplication of two matrices. $M_{x}$ has high magnitudes at pixels where the NIR and $Y$ horizontal gradients are highly correlated. On the other hand, if the horizontal gradients are not similar enough, the corresponding component in $M_{x}$ is small. $M_{y}$ has similar properties when the gradient is computed in the vertical direction. Thus, we incorporate the gradient masks to ensure that the edges of the $\mathrm{Y}$ channel contribute to the deblurring result only where gradients of NIR and Y channels are strongly correlated. 
(a) Sharp color

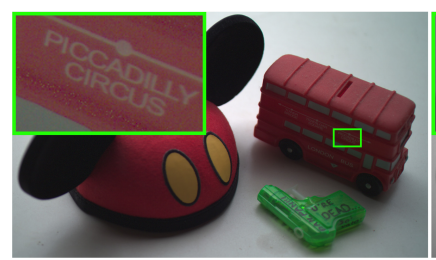

(b) Blurred NIR

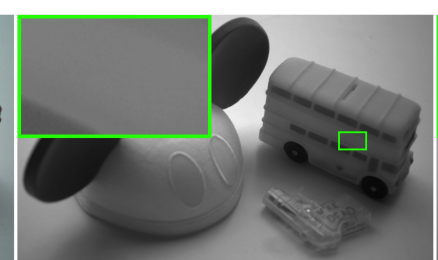

(c) Deblurred without gradient masks

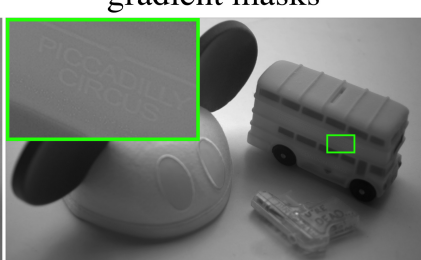

(d) Deblurred with gradient masks

Figure 3. This figure shows a pair of (a) sharp color and (b) blurred NIR images captured with the same focus settings. Image (c) is the result of deblurring guided by the color image without using gradient masks, which contains false edges not present in the NIR image. By using the gradient masks (d), the algorithm preserves the differences between color and NIR images (The differences are most noticeable while viewed on a screen).

The gradient masks are required to measure the similarity between the gradients of NIR and Y (luminance of the color image) channels. If the sharp NIR image is accessible, the similarity levels can be computed as follows:

$$
M_{x}^{0}(i, j)=1-\frac{\left|\nabla_{x} \operatorname{NIR}(i, j)-\nabla_{x} Y(i, j)\right|}{\left|\nabla_{x} \operatorname{NIR}(i, j)+\nabla_{x} Y(i, j)\right|}, \quad M_{y}^{0}(i, j)=1-\frac{\left|\nabla_{y} \operatorname{NIR}(i, j)-\nabla_{y} Y(i, j)\right|}{\left|\nabla_{y} \operatorname{NIR}(i, j)+\nabla_{y} Y(i, j)\right|},
$$

where $(i, j)$ indicate the horizontal and vertical coordinates of the pixel. Note that as the level of similarity between the gradients of NIR and $Y$ channels increases, the values of $M_{x}^{0}(i, j)$ and $M_{y}^{0}(i, j)$ increase.

However, in the deblurring problem, we do not have access to the sharp NIR image in advance. Hence, the gradient masks cannot be computed exactly as shown in (6). In addition, deblurring fails if we directly compare the gradients of the blurred NIR and the sharp Y channel. The reason is that even the edges that are inherently similar between the color and NIR representations, would have different profiles as they are blurred in NIR and sharp in the Y channel. To address this difficulty, we first deblur the $\mathrm{Y}$ channel with the estimated blur kernel:

$$
Y_{b}=k_{\sigma} * Y \text {. }
$$

and calculate the gradient masks as

$$
M_{x}(i, j)=1-\frac{\left|\nabla_{x} \operatorname{NIR}_{b}(i, j)-\nabla_{x} Y_{b}(i, j)\right|}{\left|\nabla_{x} \operatorname{NIR}_{b}(i, j)+\nabla_{x} Y_{b}(i, j)\right|}, \quad M_{y}(i, j)=1-\frac{\left|\nabla_{y} \operatorname{NIR}_{b}(i, j)-\nabla_{y} Y_{b}(i, j)\right|}{\left|\nabla_{y} \operatorname{NIR}_{b}(i, j)+\nabla_{y} Y_{b}(i, j)\right|},
$$

Comparing the gradients of blurred Y and NIR channels ensures large components for $M_{x}$ and $M_{y}$ when the edges are similar. Additionally, if in one neighborhood color and NIR edges are fundamentally uncorrelated, they are likely to look different even after blurring (unless the NIR image is severely blurred).

We discussed the significance of using gradient masks $\left(M_{x}\right.$ and $\left.M_{y}\right)$ in Section 1. Here, we illustrate this effect with one example. Figure 3 shows a pair of blurred NIR and sharp color images, and also the sharp NIR image representing the same scene (captured by re-focusing the camera for the NIR shot). Subfigure (c) is the NIR image deblurred without using gradient masks. It can be immediately observed that false edges (the text in the zoomed-in region) are introduced into the deblurred NIR image. However, when we incorporate the gradient masks into deblurring (see Fig. 3-d), the inherent differences between the color image and its NIR counterpart are preserved (the differences are more noticeable when this figure is viewed on a screen). We present more comparisons in Section 3.3.

\section{RESULTS}

\subsection{Data Acquisition and Ground-truth}

To capture the images presented in this section, we used a Canon Rebel T1i camera with a $50 \mathrm{~mm}$ kit lens. We removed the NIR-blocking filter from inside the camera, so NIR and color images were captured in two sequential shots, when visible-light blocking and NIR-blocking filters were placed in front of the lens. For every scene, we 

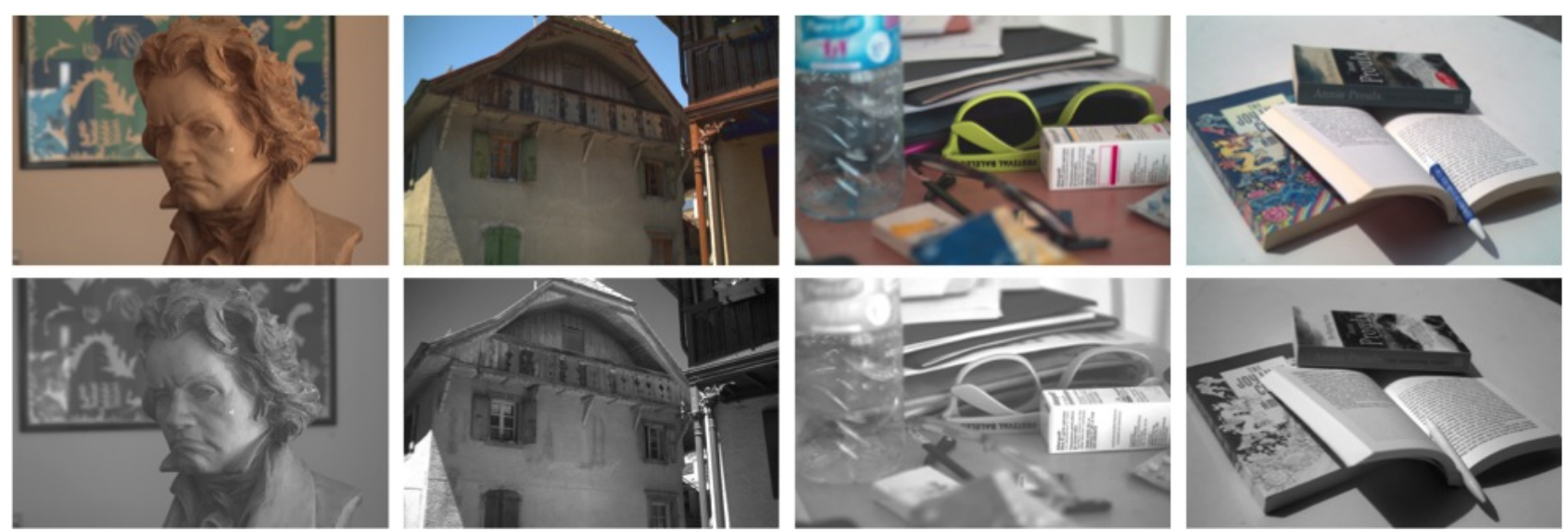

Figure 4. Four pairs from the dataset of color and NIR images used to evaluate the kernel estimation accuracy.

captured one color and two NIR images (blurred and sharp NIR images). We first adjusted the focus settings for the color image. The blurred NIR image was then captured with the same settings. Afterwards, we refocused the camera for the NIR shot, that results in the ground-truth NIR image.

We analyze the performance of our algorithm in estimating the blur kernel in subsection 3.2. We then compare the images deblurred by our algorithm with the results of Krishnan et al.'s algorithm ${ }^{14}$ in subsection 3.3.

\subsection{Blur Kernel Estimation}

In a practical scenario, when the color image is in focus and NIR is blurred (out of focus), we do not have access to the ground-truth blur kernel. Therefore, to assess our kernel estimation algorithm, we conducted a toy experiment. For this experiment, we used the color and the sharp NIR images of each scene. Some of the images used for this experiment are shown in Fig. 4. We blurred the NIR image, as modeled in (1), by a Gaussian kernel with a known standard deviation $(\sigma)$. The optimization problem $(2)$ is solved to estimate the kernel spread $(\sigma)$ by using this blurred NIR and sharp color images. Table 1 summarizes ground-truth $\sigma$ values used to blur the NIR image and the values estimated by our algorithm. The results reported in this table are averaged over 30 pairs of color and NIR images. As can be seen, the estimated values are quite close to ground-truth. Note that a small deviation from the true kernel does not greatly affect the deblurring process.

To test the kernel estimation in a more realistic case, in the second set of experiments, we used the NIR images blurred by the lens. In this experiment, we compare our blur kernel estimation method with the algorithm of Krishnan et al. ${ }^{14}$ Krishnan et al. proposed a blind deblurring method that first iteratively estimates the blur kernel from the blurred image, and at the last step uses the estimated kernel to deblur the image. Figure 5-(b) shows the image deblurred with this method. We also estimated the blur kernel with our algorithm and applied the deblurring step of Krishnan's method to produce the final image using our estimated kernel (Fig. 5-(c)). As can be seen in Fig. 5, the image deblurred using our estimated kernel contains fewer artifacts compared with the result of the blind deblurring algorithm of Krishnan et al. ${ }^{14}$ This proves the effectiveness of our algorithm in estimating the blur kernel, as both images are produced by the same deblurring technique.

\begin{tabular}{|c|c|cccccccc|}
\hline \multicolumn{2}{|c|}{ Ground-truth $\sigma$} & 3 & 4 & 5 & 6 & 7 & 8 & 9 & 10 \\
\hline \hline \multirow{2}{*}{ Estimated $\sigma$} & average & 2.88 & 3.87 & 4.82 & 5.85 & 6.81 & 7.78 & 8.83 & 9.55 \\
\cline { 2 - 9 } & std. & 0.25 & 0.32 & 0.38 & 0.44 & 0.61 & 0.65 & 0.77 & 0.77 \\
\hline
\end{tabular}

Table 1. Blur kernel estimation: for each ground truth $\sigma$ value that is used to blur the NIR images, we report the estimated $\sigma$ obtained by using the blurred NIR and sharp color images. The experiment is conducted for 30 pairs of images. 
(a) Blurred NIR image

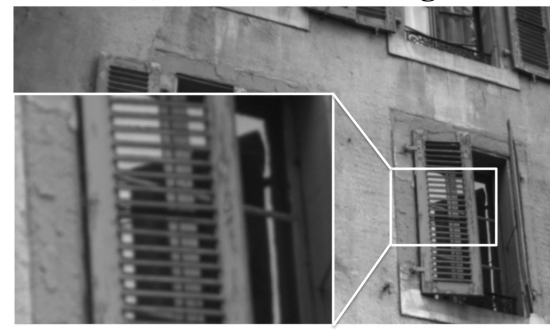

(b) Result of Krishnan et al.

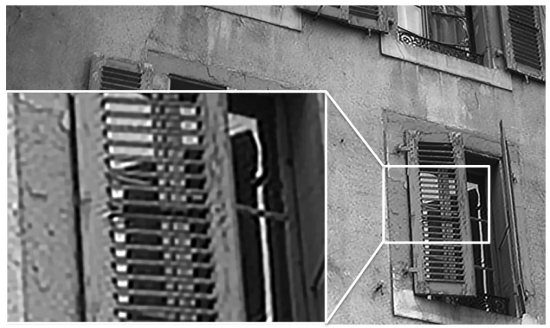

(c) Result of Krishnan et al. with our kernel

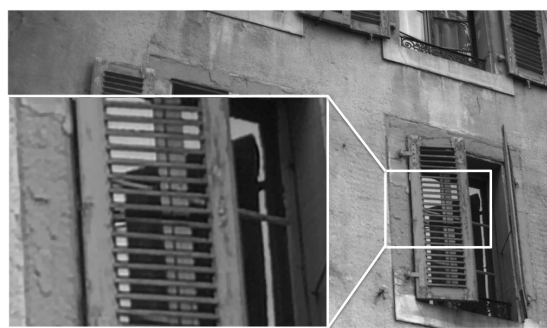

Figure 5. (a) The blurred NIR image, (b) the result of the blind deblurring algorithm of Krishnan et al., ${ }^{14}$ and (c) the result of the deblurring step of Krishnan's algorithm using our estimated kernel.

\subsection{Guided Deblurring}

In this subsection, we analyze the performance of our guided deblurring algorithm. We first study the importance of using gradient masks in deblurring. Figure 6-(c) shows the results of our algorithm when no gradient mask is used in the optimization (see (4) and (5)). Images of Fig. 6-(d) are the outputs of the algorithm when $M_{x}$ and $M_{y}$ matrices are employed. As shown in the second and fifth rows of this figure (highlighted by boxes with green borders), without the gradient mask, false edges appear in the deblurred NIR image. We observe that the designed gradient masks are very effective in solving this issue and in preventing the optimization from introducing false edges into the deblurred image.

The inevitable consequence of using masks is that the fine details of the color image contribute less to the deblurred image, even when the gradients are highly correlated (see rows 3 and 6 of Fig. 6). This is mainly because the masks are computed using the gradients of blurred NIR and blurred Y channels. As a result, these masks provide only an approximation of the true similarity map between the latent sharp NIR and sharp Y channels.

In the next experiment, we study the performance of the deblurring step when different prior terms for the sharp NIR image, $\operatorname{Pr}(\mathrm{NIR})$ in (4), are explored. Figure 7 provides some examples for this comparison. The first and second columns of this figure are the input color and NIR images. Figure 7-(c) shows the deblurring results when the popular TV (total variation) regularization is used as $\operatorname{Pr}(\mathrm{NIR})$. In this case, the following optimization is solved to deblur NIR:

$$
\mathrm{NIR}^{\star}=\operatorname{argmin}\left\|\mathrm{NIR}_{b}-k_{\sigma} * \mathrm{NIR}\right\|_{F}+\lambda\|\nabla \mathrm{NIR}\|_{1} .
$$

The deblurring step in the Krishnan et al. method $^{14}$ is used to produce the images of Fig. 7-(d). This algorithm exploits a more sophisticated prior, namely a normalized sparsity of image gradients. Images of subfigure (e) are the results of our proposed algorithm when the gradients of the color image are used to constrain the problem (see equations (4) and (5)). We also studied the effect of adding a TV regularizer to our deblurring optimization problem as follows:

$$
\mathrm{NIR}^{\star}=\operatorname{argmin}\left\|\mathrm{NIR}_{b}-k_{\sigma} * \mathrm{NIR}\right\|_{F}+\lambda_{1}\left(\left\|\nabla_{x} \mathrm{NIR}-M_{x} \odot \nabla_{x} Y\right\|_{F}+\left\|\nabla_{y} \mathrm{NIR}-M_{y} \odot \nabla_{y} Y\right\|_{F}\right)+\lambda_{2}\|\nabla \mathrm{NIR}\|_{1} .
$$

The results are shown in Fig. 7-(f). For each image, the blur kernel estimated with our method is used with different deblurring techniques to generate the different results in Fig. 7.

These results clearly show that using a regularization term specific to each blurred NIR image (the gradients of the sharp color image in our algorithm) is much more effective than exploiting general distributions. Our algorithm results in more accurate edges and significantly less noticeable artifacts, compared to other techniques presented in Fig. 7-(c) and (d). Moreover, adding the TV regularization term does not improve the performance of our algorithm (compare subfigures (e) and (f)). 


\section{CONCLUSION}

We have proposed a novel algorithm that reduces the distortions caused by axial chromatic aberration in the joint acquisition of color and NIR images. Our algorithm first estimates the blur kernel by comparing the gradient of the blurred NIR image with the sharp color image. In the next step, being guided by the edges in the luminance channel of the color image and by using the estimated kernel, the algorithm deblurs the NIR image. The key component of our deblurring method is the use of gradient masks that effectively prevent the deblurred NIR image from inheriting false edges from the color image.

In this paper, we assumed that the blur kernel is spatially invariant. However, the lens blur kernel depends on the depth of objects in the scene. Hence, in a general case, the blur kernel varies spatially. Estimating spatially variant kernels and adapting the proposed deblurring algorithm to use such kernels are part of our future work.

\section{Acknowledgments}

This work was supported by the Hasler Foundation under grant number 12100 and the Qualcomm Innovation Fellowship program.

\section{REFERENCES}

1. Yan, Q., Shen, X., Xu, L., Zhuo, S., Zhang, X., Shen, L., and Jia, J., "Cross-field joint image restoration via scale map," in [International Conference on Computer Vision (ICCV)], (2013).

2. Feng, C., Zhuo, S., Zhang, X., Shen, L., and Süsstrunk, S., "Near-infrared guided color image dehazing," in [IEEE International Conference on Image Processing (ICIP)], (2013).

3. Rüfenacht, D., Fredembach, C., and Süsstrunk, S., "Automatic and accurate shadow detection using nearinfrared information," IEEE Transactions on Pattern Analysis and Machine Intelligence (TPAMI) 36, 1672-1678 (2014).

4. Salamati, N., Germain, A., and Süsstrunk, S., "Removing shadows from images using color and nearinfrared," in [IEEE International Conference on Image Processing (ICIP)], (2011).

5. Gunawardane, P., Malzbender, T., Samadani, R., McReynolds, A., Gelb, D., and Davis, J., "Invisible light: Using infrared for video conference relighting," in [IEEE International Conference on Image Processing $($ ICIP)], (2010).

6. Lu, Y. M., Fredembach, C., Vetterli, M., and Süsstrunk, S., "Designing color filter arrays for the joint capture of visible and near-infrared images," in [IEEE International Conference on Image Processing (ICIP)], (2009).

7. Sadeghipoor, Z., Lu, Y., and Süsstrunk, S., "A novel compressive sensing approach to simultaneously acquire color and near-infrared images on a single sensor," in [IEEE International Conference on Acoustics, Speech, and Signal Processing (ICASSP)], (2013).

8. Luo, X. Y., Zhang, J., and Dai, Q. H., "Hybrid fusion and demosaicing algorithm with near-infrared image," in [SPIE Proceedings: Multisensor, Multisource Information Fusion: Architectures, Algorithms, and Applications], (2014).

9. Kang, S. B., "Automatic removal of chromatic aberration from a single image," in [IEEE Conference on Computer Vision and Pattern Recognition (CVPR)], (2007).

10. Tisse, C., Nguyen, H. P., Tessiéres, R., Pyanet, M., and Guichard, F., "Extended depth-of-field (EDoF) using sharpness transport across colour channels," in [SPIE Proceedings: Novel Optical Systems Design and Optimization XI], (2008).

11. Chung, S. W., KIM, B. K., and Song, W. J., "Detecting and eliminating chromatic aberration in digital images," in [IEEE International Conference on Image Processing (ICIP)], (2009).

12. Heide, F., Rouf, M., Hullin, M. B., Labitzke, B., Heidrich, W., and Kolb, A., "High-quality computational imaging through simple lenses," ACM Transactions on Graphics (TOG) 32 (2013).

13. Levin, A., Weiss, Y., Durand, F., and Freeman, W., "Understanding and evaluating blind deconvolution algorithms," in [IEEE Conference on Computer Vision and Pattern Recognition (CVPR)], (2009).

14. Krishnan, D., Tay, T., and Fergus, R., "Blind deconvolution using a normalized sparsity measure," in [IEEE Conference on Computer Vision and Pattern Recognition (CVPR)], (2011). 
15. Kee, E., Paris, S., Chen, S., and Wang, J., "Modeling and removing spatially-varying optical blur," in [IEEE International Conference on Computational Photography (ICCP)], (2011).

16. Shen, C., Hwang, W., and Pei, S., "Spatially-varying out-of-focus image deblurring with 11-2 optimization and a guided blur map," in [IEEE International Conference on Acoustics, Speech, and Signal Processing (ICASSP)], (2012).

17. He, K., Sun, J., and Tang, X., "Guided image filtering," IEEE Transactions on Pattern Analysis and Machine Intelligence (TPAMI) 35, 1397-1409 (2013).

18. Yuan, L., Sun, J., Quan, L., and Shum, H., "Image deblurring with blurred/noisy image pairs," $A C M$ Transactions on Graphics (TOG) $\mathbf{2 6}$ (2007).

19. Zhang, H., Wipf, D., and Zhang, Y., "Multi-image blind deblurring using a coupled adaptive sparse prior," in [IEEE Conference on Computer Vision and Pattern Recognition (CVPR)], (2013).

20. Tatian, B., "Method for obtaining the transfer function from the edge response function," Journal of the Optical Society of America 55, 1014-1019 (1965).

21. Hwang, S., Kim, K., Shin, J., Paik, J., Abidi, B., and Abidi, M., "Real-time digital autofocusing using a priori estimated set of PSFs," in [SPIE Proceedings: Real-Time Imaging VIII], (2004).

22. Bioucas-Dias, J. M., Figueiredo, M. A. T., and Oliveira, J. P., "Total variation-based image deconvolution: A majorization-minimization approach," in [IEEE International Conference on Acoustics, Speech, and Signal Processing (ICASSP)], (2006).

23. Krishnan, D. and Fergus, R., "Fast image deconvolution using hyper-laplacian priors," in [NIPS], (2009).

24. Joshi, N., Zitnick, C., Szeliski, R., and Kriegman, D. J., "Image deblurring and denoising using color priors," in [IEEE Conference on Computer Vision and Pattern Recognition (CVPR)], (2009). 
(a) Color image

(b) Blurred NIR image

(c) Our method without gradient masks

(d) Our method with gradient masks

(e) Ground-truth NIR

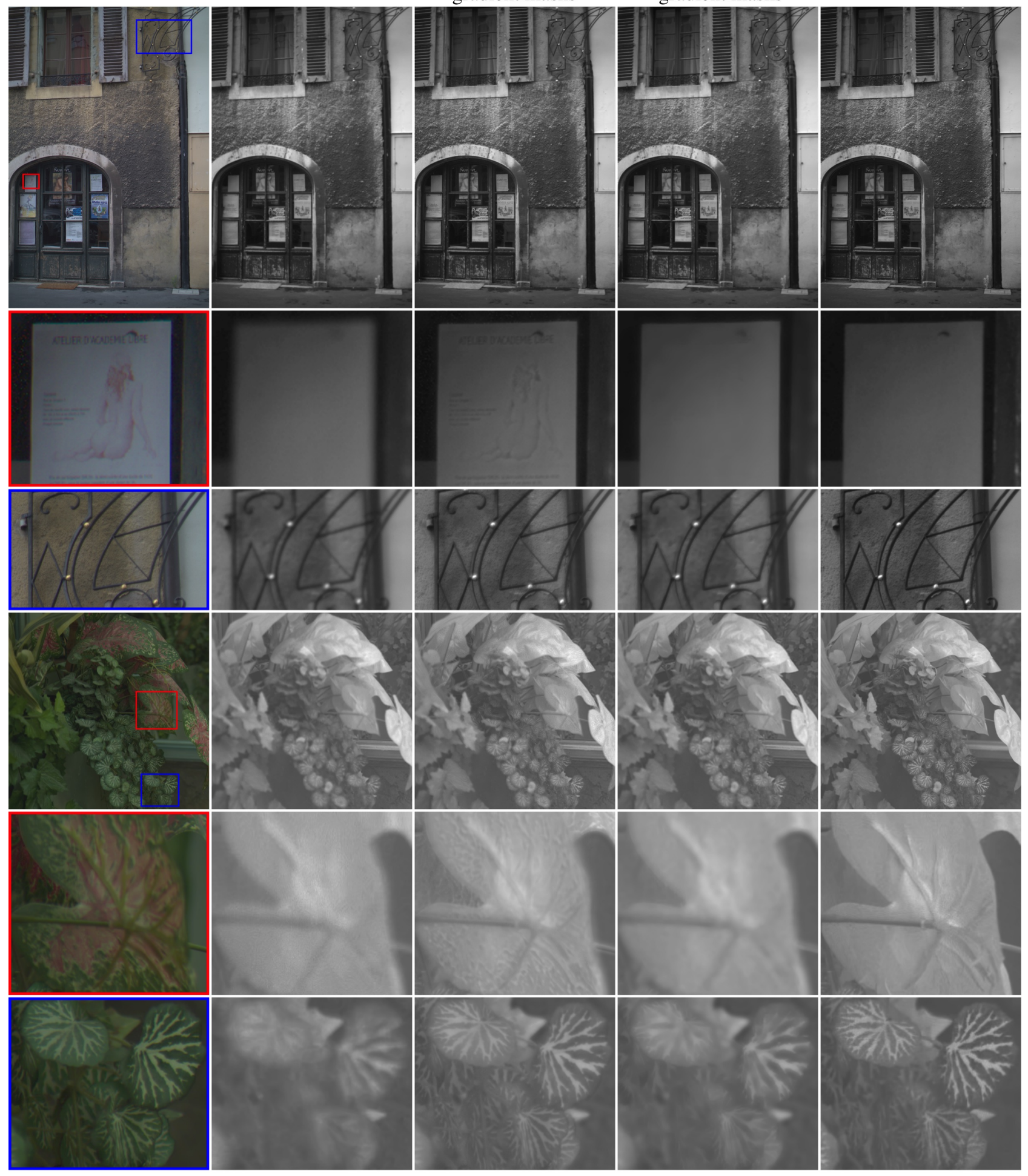

Figure 6. (a) and (b) the color and NIR images captured with the same focus settings, (c) the results of our method when no gradient mask is used, (d) our algorithm's results by using the gradient mask, and (e) the sharp NIR images captured by changing the focus settings. The differences are more easily seen when this figure is viewed on a screen. 


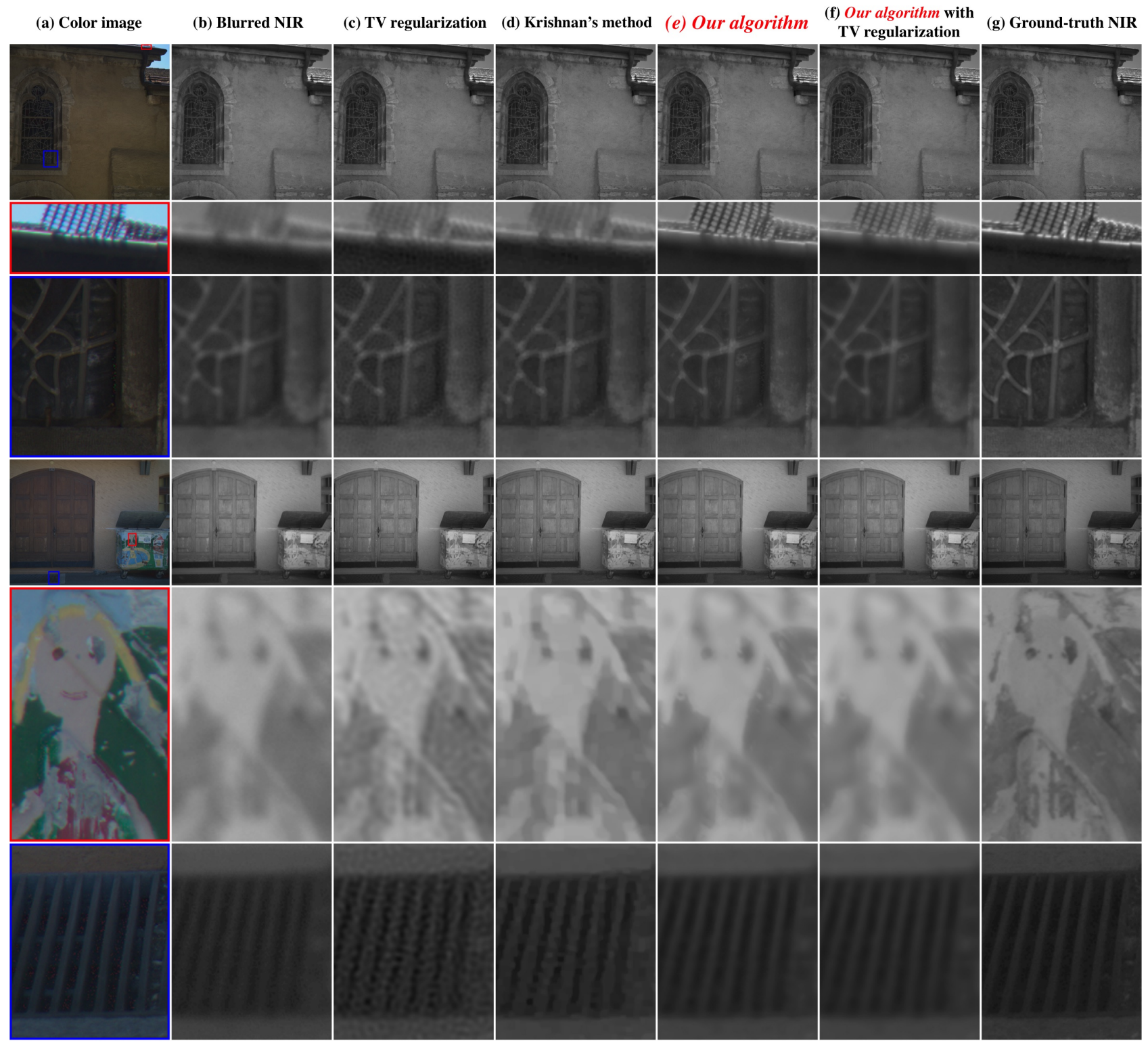

Figure 7. (a) the color and (b) NIR images captured with the same focus settings, (c) the results of deblurring with TV regularization, (d) Krishnan's method deblurring output, ${ }^{14}$ (e) the results of our algorithm, (f) images deblurred by our algorithm when a TV regularization term is added, and (f) the sharp NIR images captured by changing the focus settings. The differences are more easily seen when the figure is viewed on a screen. 